\section{Australian Journal of \\ Crop Science}

AJCS

ISSN:1835-2707

AJCS 14(01):28-35 (2020)

doi: 10.21475/ajcs.20.14.01.p1704

\title{
Meteorological conditions and their associations with the productive performance of wheat
}

\author{
Thomas Newton Martin*, José Eduardo Minussi, Jessica Deolinda Leivas Stecca, Giovani Mathias Burg, \\ Marlo Bison Pinto
}

\author{
Department of Crop Science, Universidade Federal de Santa Maria, Santa Maria, RS, Brazil
}

\section{*Corresponding author: martin.ufsm@gmail.com}

\begin{abstract}
This study aimed at assessing the effect of the photothermal quotient, meteorological variables and their effects on the grain production and yield of the wheat cultivars in terms of hectoliter mass in Santa Maria, Rio Grande do Sul state, Brazil. The experiments were carried out in the same experimental area in different years. Four experiments were performed in the experimental field during the agricultural years of 2013 (10 cultivars), 2014 (16 cultivars), 2015 (15 cultivars) and 2016 (15 cultivars) with the principal cultivars in the Rio Grande do Sul state, during each of these years. The meteorological factors (mean air temperature, solar radiation, insolation and accumulated precipitation) showed wide fluctuations over these years. They induced instability in the production. The grain yield and the hectoliter mass were compared in each of the agricultural years (Scott-Knott, $\alpha \leq 0.05$ ) and are related in dispersion plots according to the climatic variables for the set of years and cultivars (regression analysis). The available solar radiation (number of sunshine hours), quality (photothermal quotient) and average air temperature were the determinant factors for wheat productivity. However, the hectoliter mass was influenced more by the effect of genetic variability, lack of precipitation during crop maturation, and photothermal quotient around the time of anthesis. Wheat crop investments can be expanded to maximize wheat grain yield when average cycle temperatures remain near $16.5{ }^{\circ} \mathrm{C}$, high solar radiation and low rainfall. Better quality trains (hectoliter mass) will be obtained when smaller precipitations are observed at harvest and higher photothermal quotient.
\end{abstract}

Keywords: Heat stroke; rainfall; solar radiation; temperature.

\section{Introduction}

From a global perspective, wheat is the second most produced cereal, with 725 million tons being the annual yield (USDA, 2016). In 2016, Brazil recorded wheat output of 6.72 million tons. At present, Brazil is necessary to import $45 \%$ of its demand as the national wheat yield is less than the domestic demand (Conab, 2016). The state of Rio Grande do Sul has favorable climatic conditions for the cultivation of wheat (Mapa, 2017), registering 15ㅇ C average temperature and $840 \mathrm{~mm}$ accumulated during the growing season (between May and October). However, in the last five harvests, this state's productivity ranged from $1330 \mathrm{~kg}$ $\mathrm{ha}^{-1}$ to $3214 \mathrm{~kg} \mathrm{ha}^{-1}$, and this variability are attributed to meteorological conditions prevailing during the crop seasons.

The main meteorological variables which influence the growth, development, production and quality of the spring wheat grains are the availability of solar radiation, particularly around the flowering season, and average temperatures between 15 and 25ㅇ (Monteiro et al., 2009). Air temperature and photoperiod controlling the duration of the stages and developmental cycle of the spring wheat (Alberto et al., 2009), and their development differ according the cultivar and the developmental phase of the crop (Lizana and Calderini, 2012). The productivity potential of the wheat crop indicates a relationship existing between the number of spikes, number of spikelets per spike, grain mass and hectoliter mass. The relationship between air temperature, solar radiation, quotient phototermal (PQ), photoperiod and precipitation may change the photosynthetic production in each of the stages (Silva et al., 2014; Lizana and Calderini, 2012). The PQ was determined according to the model proposed by Fischer (1985), where $P Q=R S /(T m e d-T b)$. Here, $P Q$ is the daily photothermal quotient $\left(\mathrm{MJ} \mathrm{m}^{-2}\right.$ day $\left.{ }^{\circ} \mathrm{C}\right), \mathrm{RS}$ is the global solar radiation (MJ $\mathrm{m}^{-2}$ day), Tmed is the mean daily temperature $\left({ }^{\circ} \mathrm{C}\right)$ and $\mathrm{Tb}$ is the basal temperature.

This quotient photothermal can be used to predict or explain wheat production (Kumar et al., 2016) and frost risk to identify suitable sowing dates for wheat (Silva et al., 2014), estimation of wheat grain yield (Ratjen et al., 2012). The PQ can be used to identify sowing times and regions that maximize grain yield potential (Nalley et al., 2009; Silva et al., 2014). The sensitive stages of wheat to temperature and water stress were calculated using quotient photothermal in Australia (Dreccer et al., 2018). The research for characterization of spatial and temporal combinations of climatic factors affecting yields was conducted using empirical model applied to the French barley belt (Beillouin et al., 2018). Fischer (2016) investigated the relationship between the duration of the vegetative period (sowing to 
the anthesis) and the productivity components in semidwarf wheat cultivars in Mexico using the PQ.

Therefore, further research is crucial to understand the physiological, morphological and productive behaviors of the wheat cultivars and their responses to a variety of combinations of temperature, solar radiation and precipitation (Silva et al., 2014). However, none of the recent studies evaluated the relationship between the photothermal quotient and production of spring wheat cultivars under the climatic conditions of Santa Maria, Rio Grande do Sul. Therefore, this study attempted to determine the relation of the photothermal quotient, meteorological variables and their relationships with the grain productivity and hectoliter mass of the wheat cultivars.

\section{Results and discussion}

During the growing seasons, the meteorological data revealed that the experiments were not exposed to water deficiency conditions (Fig 1).

Significant differences were noted for both the variables evaluated (Table 1 ) during all the four agricultural years. The hectoliter mass (MH) in 2016, signified differences in the genetic composition of the cultivars, and reliance on the cultivar as well. Thus, the cultivars expressed different behaviors during each climatic condition.

In 2013, while all the cultivars revealed a high yield exceeding the national average, the Itaipu and Quartzo cultivars gave the highest grain productivity at $3728.83 \mathrm{~kg}$ $\mathrm{ha}^{-1}$ and $3576.49 \mathrm{~kg} \mathrm{ha}^{-1}$, respectively, because the Santa Maria region experienced favorable weather conditions during the growing season. Considering the hectoliter mass, most of the cultivars did not differ beyond $5 \%$, with the exception of cultivar Fundacep Bravo which displayed a lower $\mathrm{MH}$ value than did the other cultivars ( $\mathrm{MH} 71.70)$. In 2014, the following cultivars, CEP08180 (2276.70 kg ha-1), FUNDACEP Bravo (2143.94 $\left.\mathrm{kg} \mathrm{ha}^{-1}\right)$, CEP07244 $(2065.32 \mathrm{~kg}$ $\left.\mathrm{ha}^{-1}\right)$, TEC Frontale (2036.15 kg ha-1), Quartz (2004.71 kg ha$\left.{ }^{1}\right)$, CEP08144 (1996.21 $\mathrm{kg} \mathrm{ha}^{-1}$ ), Fundacep Horizonte (1904.30 $\mathrm{kg} \mathrm{ha}^{-1}$ ) and TBIO Itaipu (1866.52 $\mathrm{kg} \mathrm{ha}^{-1}$ ) gave the highest $\mathrm{MH}$ values. The cultivar Ametista showed a low grain yield value (1416.02 $\mathrm{kg} \mathrm{ha}^{-1}$ ) but a high $\mathrm{MH}$ value (72.74), indicating high industrial quality characteristic of this cultivar. During this year, all the cultivars in the experiment gave yields exceeding the state average but less than the national average. In 2015, most of the cultivars showed productivity exceeding the state and national averages, with the most productive being the cultivars BRS327 (3056.98 kg $\mathrm{ha}^{-1}$ ) and TBIO Toruk (2943.00 $\mathrm{kg} \mathrm{ha}^{-1}$ ). Only the BRS327 cultivar showed high $\mathrm{MH}$ values. The cultivar Ametista revealed agronomic performance that enabled the inference that variations in the quality of grain produced was possible under different environmental conditions.

Most cultivars in the experiment (2016) showed more than $4500 \mathrm{~kg} \mathrm{ha}^{-1}$ in yield, excepting for the BRS 331 cultivar. The average in this experiment was $5195.15 \mathrm{~kg} \mathrm{ha}^{-1}$, which was 1.6 times higher than the average values of the state and nation. Similarly, the hectoliter mass values were too high, being greater than that of the other years, indicating of the favorable meteorological conditions, during the critical developmental phases of the culture, particularly in the anthesis and grain filling stages, which produced the expression of the potential of productivity by the wheat cultivars.

From a comparison of the results of the cultivars that were repeated in more than one agricultural year (Table 2), it is evident that the highest productivity and $\mathrm{MH}$ values were observed for 2013 and 2016. This indicates the favorable climatic factors during these harvests, as the RS state revealed the highest historical yield in the year 2016 (3214 $\mathrm{kg} \mathrm{ha}^{-1}$ ). When the same cultivars that sown in 2013 were sown again in 2014, presented $46 \%$ lower mean productivity (1669.64 $\mathrm{kg} \mathrm{ha}^{-1}$ ), with 69.37 as the mean $\mathrm{MH}$ value, which was 6 points lower than the yield of 2013 (3189.39 kg ha-1 and $\mathrm{MH}$ 75.66).

When the same cultivars were repeated in 2014 and 2015, they showed the highest productivity values for the year 2015 (2386.88 $\mathrm{kg} \mathrm{ha}^{-1}$ on average), with $36 \%$ higher yield than for 2014 (1522.1 $\mathrm{kg} \mathrm{ha}^{-1}$ on average), while the $\mathrm{MH}$ values remained the same for both the agricultural years. When these cultivars were repeated in 2015 and 2016, the average yield of $2015\left(2599.20 \mathrm{~kg} \mathrm{ha}^{-1}\right)$ doubled in 2016 ( $5273.47 \mathrm{~kg} \mathrm{ha}^{-1}$ ), and the $\mathrm{MH}$ in 2016 rose by 11 points compared with that in 2015.

The data on the crop yield in 2013, 2014, 2015 and 2016 revealed that the agricultural year of 2016 experienced the best climate during the developmental cycle of the wheat crop, in terms of the factors like temperature, solar radiation and precipitation. Besides, the high yield values were partly due to the genetic gain, because the new cultivars have been adopted. Correlation studies among the agrometeorological indicators and grain yield of the wheat had been performed earlier in the northern parts of RS (Mota, 1998; Nalley et al., 2009; Silva et al., 2014). The factors such as duration of the leaf wetting period in October, total insolation in September, and frost damage index in September and number of rainfall days in November were studied. Solar radiation and temperature are the strong forces which drive the developmental rate and wheat production, diversely steering the plant processes, although the findings of their collective productivity are evident from the photothermal quotient (Ahmed et al., 2010).

Among the meteorological factors, the average temperature of the crop cycles represents an effect that is inversely proportional to wheat productivity (Fig 2). The findings of this experiment concur with those of Cargnin et al. (2006) and Semenov et al. (2014) who reported that the number of grains exerts a negative linear relationship with temperature, adversely affecting the yield by accelerating the cycle during the critical 30-day period, around the time of anthesis (Monteiro, 2009; Ratjen et al., 2012). Scheeren et al. (2007) reported an ideal temperature of 15 and $20 \circ \mathrm{C}$ during pickling, while between 20 and $25 \circ \mathrm{C}$ is ideal for leaf development. Temperature responses act as determinants for the plant processes, like enzymatic activities and photosynthesis, whose rates and efficiencies are temperature dependent. Every plant displays different temperature sensitivity during the morphological and phenological stages (Porter and Gawith, 1999).

The agricultural year of 2013 registered the lowest average temperature $\left(16.29^{\circ} \mathrm{C}\right)$ with 2016 following in second place $\left(16.83^{\circ} \mathrm{C}\right)$, with average yields of $3206.48 \mathrm{~kg} \mathrm{ha}^{-1}$ and $5195.28 \mathrm{~kg} \mathrm{ha}^{-1}$, respectively. The year of 2014, however, experienced temperature of $18.34^{\circ} \mathrm{C}$ on average and the 

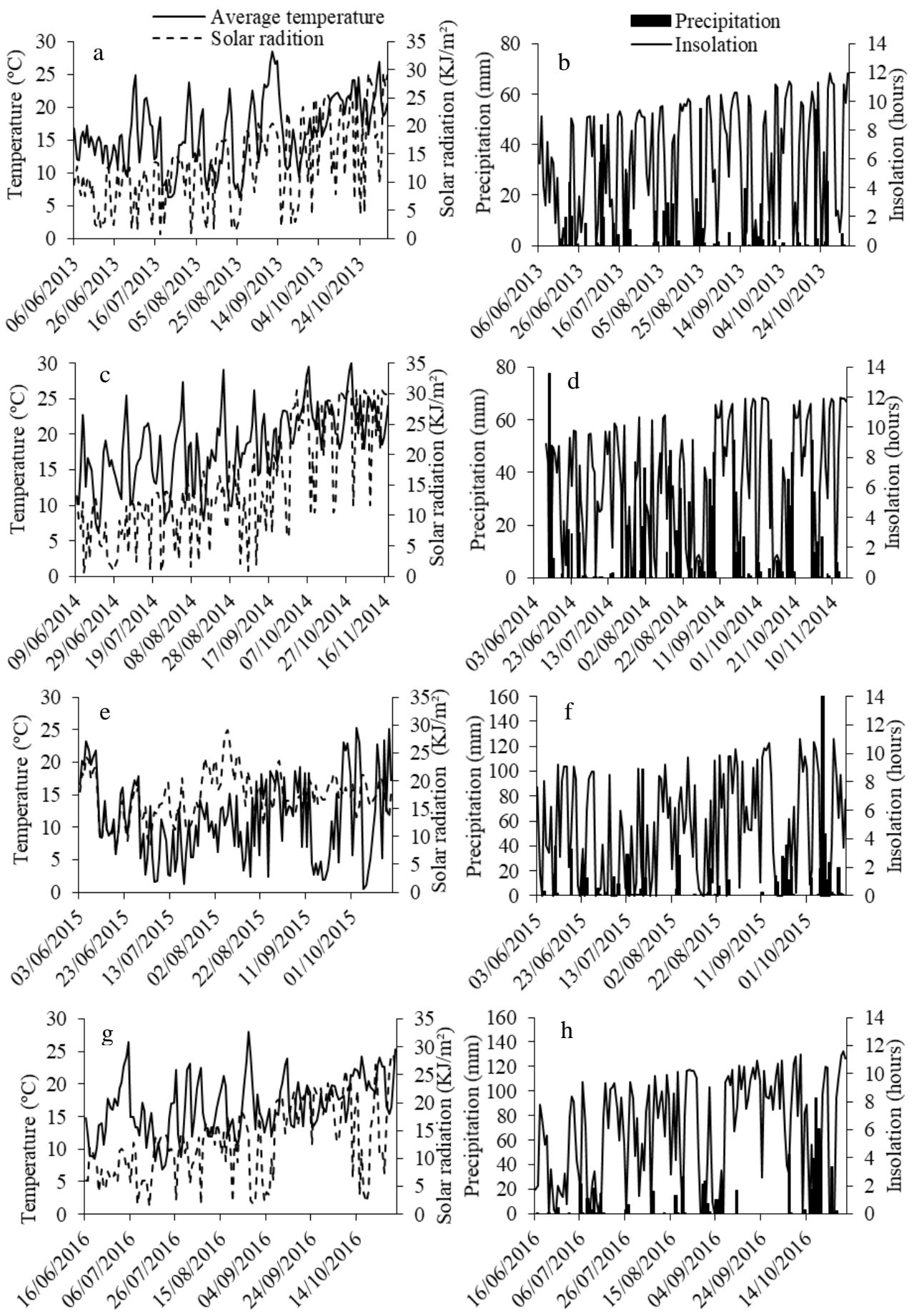

Fig 1. Mean air temperature $(\stackrel{\circ}{-C})$ and solar radiation $\left(\mathrm{KJ} \cdot \mathrm{m}^{-2}\right)\left(\mathrm{a}, \mathrm{c}, \mathrm{e}\right.$ and $\mathrm{g}$ ), and precipitation ( $\mathrm{mm}$ day $\left.\mathrm{y}^{-1}\right)$ and insolation (hours) (b, d, $f$ and $h$ ) during the Santa Maria experiments in the agricultural years 2013, 2014 and 2015 and 2016.

Table 1. Values of the mean square of the ANOVA for grain yield (PG) $\left(\mathrm{kg} \mathrm{ha}^{-1}\right)$ as well as hectoliter mass (MH) $\left(\mathrm{kg} \mathrm{hl}^{-1}\right)$ values for the wheat cultivars evaluated during the agricultural years between 2013 and 2016.

\begin{tabular}{|c|c|c|c|c|c|c|c|c|c|}
\hline \multirow{3}{*}{$\begin{array}{l}\text { Source } \\
\text { variation }\end{array}$} & \multirow{3}{*}{ of } & \multicolumn{8}{|l|}{ Mean Square } \\
\hline & & \multicolumn{2}{|l|}{2013} & \multicolumn{2}{|l|}{2014} & \multicolumn{2}{|l|}{2015} & \multicolumn{2}{|l|}{2016} \\
\hline & & PG & $\mathrm{MH}$ & $P G$ & $\mathrm{MH}$ & $P G$ & $\mathrm{MH}$ & PG & $\mathrm{MH}$ \\
\hline Cultivars & & $505812.82^{*}$ & $13.17^{*}$ & 441717.84* & $38.39 *$ & 537553.56* & $56.65^{*}$ & 982217.11* & $9.84^{\text {ns }}$ \\
\hline Block & & 70221.91 & 1.33 & 5742.13 & 1.71 & 8536.80 & 6.15 & 108093.66 & 3.18 \\
\hline Error & & 31220.32 & 2.31 & 31744.54 & 4.32 & 60281.43 & 3.22 & 207111.11 & 4.87 \\
\hline CV (\%) & & 5.61 & 1.99 & 10.18 & 2.99 & 9.79 & 2.70 & 8.76 & 2.87 \\
\hline
\end{tabular}




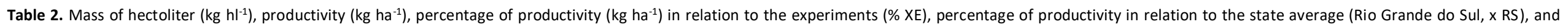
percentage of productivity in relation to the national average, (\% x BR) of the wheat cultivars cultivated during the agricultural years of 2013, 2014, 2015 and 2016 in Santa Maria, RS.

\begin{tabular}{|c|c|c|c|c|c|c|c|c|c|}
\hline Cultivars/2013 & Soure & Cycle(t) & $\begin{array}{l}\begin{array}{l}M H \\
\left(\mathrm{~kg} \mathrm{~h}^{-1}\right)\end{array} \\
\end{array}$ & & $\begin{array}{l}\begin{array}{l}\text { Productivity } \\
\left(\mathrm{kg} \mathrm{ha}^{-1}\right)\end{array} \\
\end{array}$ & & \%XE* & $\% \mathrm{XRS}^{* *}$ & $\% \times \mathrm{XR}^{* * *}$ \\
\hline TBBO Itaipu & Biotrigo & $\begin{array}{l}\text { Medium } \\
\text { Medium }\end{array}$ & 76.55 & $a^{*}$ & 3728.83 & a & $\begin{array}{l}116.29 \\
11154\end{array}$ & 121.86 & 149.03 \\
\hline $\begin{array}{l}\text { Quartzo } \\
\text { Mirante }\end{array}$ & $\begin{array}{l}\text { OR Sementes/Biotrigo } \\
\text { OR Sementes }\end{array}$ & Medium & 76.25 & a & $\begin{array}{l}3576.49 \\
30360\end{array}$ & a & 111.54 & 116.88 & $\begin{array}{l}142.95 \\
13725\end{array}$ \\
\hline 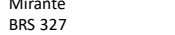 & $\begin{array}{l}\text { RR Sementes//Biotrigo } \\
\text { EMBRAPA Trigo }\end{array}$ & $\begin{array}{l}\text { Precocious } \\
\text { Precocious }\end{array}$ & $\begin{array}{l}76.13 \\
7723\end{array}$ & a & $\begin{array}{l}3436.60 \\
326081\end{array}$ & b & $\begin{array}{l}107.18 \\
10169\end{array}$ & $\begin{array}{l}112.31 \\
10656\end{array}$ & $\begin{array}{l}137.35 \\
13033\end{array}$ \\
\hline 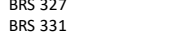 & $\begin{array}{l}\text { EMBAAPA Trigo } \\
\text { EMBRAPA Trigo }\end{array}$ & $\begin{array}{l}\text { Precocoious } \\
\text { Super-precocious }\end{array}$ & $\begin{array}{l}77.23 \\
77.62\end{array}$ & a & $\begin{array}{l}3260.81 \\
3159.96\end{array}$ & $\begin{array}{l}\mathrm{o} \\
\mathrm{c}\end{array}$ & $\begin{array}{l}10.09 \\
98.55\end{array}$ & 10320 & 12630 \\
\hline $\begin{array}{l}\text { BRS Guamirim } \\
\text { BRS Gu }\end{array}$ & EMBRAPA Trigo & $\begin{array}{l}\text { Sper-precococious } \\
\text { Precocious }\end{array}$ & 77.75 & a & $\begin{array}{l}3158.96 \\
3084\end{array}$ & $c$ & $\begin{array}{l}98.50 \\
96.07\end{array}$ & $\begin{array}{l}100.66 \\
100\end{array}$ & $\begin{array}{l}120.30 \\
123.12\end{array}$ \\
\hline FUNDACEP Bravo & Fundacep & Medium & 71.70 & b & 2946.85 & c & 91.90 & 96.30 & 117.78 \\
\hline TBIO Pioneiro & Biotrigo & Medium & 75.52 & a & 2925.49 & c & 91.24 & 95.60 & 116.93 \\
\hline $\begin{array}{l}\text { TEC } 6219 \\
\text { FUDPACP Rázes }\end{array}$ & $\begin{array}{l}\text { CCGL-TEC } \\
\text { Fundacep }\end{array}$ & $\begin{array}{l}\text { Precocious } \\
\text { Precogious }\end{array}$ & $\begin{array}{l}76.70 \\
7779\end{array}$ & $\mathrm{a}$ & $\begin{aligned} 2742.93 \\
\end{aligned}$ & d & 85.54 & 89.64 & 109.63 \\
\hline $\begin{array}{l}\text { FUDDACEP Raizes } \\
\text { Média }\end{array}$ & & & $\begin{array}{l}77.97 \\
76.34 \\
\end{array}$ & & $\begin{array}{l}\frac{2633.51}{3149.41} \\
\end{array}$ & d & & & 105.35 \\
\hline Cultivars/2013 & Soure & Cycle & $\begin{array}{l}\mathrm{MH} \\
\left(\mathrm{kghh} \mathrm{h}^{-1}\right)\end{array}$ & & $\begin{array}{l}\text { Productivity } \\
\left(\mathrm{kg} \mathrm{hat}^{2}\right)\end{array}$ & & $\%$ \%E & \%XRS** & $\% \times B R^{* *}$ \\
\hline Cultivars/2014 & Holder & Cycle & $\mathrm{MH}$ & & Productivity & & $\% \times E$ & $\% \times \mathrm{RS}^{* *}$ & $\% \times B R^{* *}$ \\
\hline CEP 08180 & & & 73.80 & a & 2276.70 & $\mathrm{a}$ & 130.09 & 171.18 & 105.16 \\
\hline FUNDACEP Bravo & Fundacep & Medium & 68.49 & b & $\begin{array}{l}2143.94 \\
20652\end{array}$ & a & 122.51 & 161.20 & 99.03 \\
\hline $\begin{array}{l}\text { CEE 0 72444 } \\
\text { TEC Frontrale }\end{array}$ & CCGL-TEC & Medium & $\begin{array}{l}68.79 \\
6.93\end{array}$ & $\begin{array}{l}b \\
b \\
b\end{array}$ & $\begin{array}{l}2065.32 \\
203.15\end{array}$ & a & $\begin{array}{l}118.01 \\
111.35\end{array}$ & $\begin{array}{l}155.29 \\
15309\end{array}$ & $\begin{array}{r}95.40 \\
94.05\end{array}$ \\
\hline 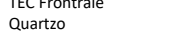 & OR Sementes/Biotrigo & $\begin{array}{l}\text { Meacum } \\
\text { Medium }\end{array}$ & $\begin{array}{l}6.9 .13 \\
74.09\end{array}$ & $\begin{array}{l}\mathrm{b} \\
\mathrm{a}\end{array}$ & $\begin{array}{l}2030.15 \\
2004.71\end{array}$ & $\begin{array}{l}\mathrm{a} \\
\mathrm{a}\end{array}$ & $\begin{array}{l}111.35 \\
114.55 \\
\end{array}$ & $\begin{array}{l}153.09 \\
150.73\end{array}$ & $\begin{array}{l}94.05 \\
92.60\end{array}$ \\
\hline $\begin{array}{l}\text { Duartzo } \\
\text { CEP } 08144\end{array}$ & OR sementess/liotingo & & $\begin{array}{l}17.499 \\
69.30\end{array}$ & $\begin{array}{c}\mathrm{a} \\
\mathrm{b}\end{array}$ & $\begin{array}{l}2004 . / 1 \\
1996.21\end{array}$ & a & $\begin{array}{l}114.55 \\
114.06\end{array}$ & $\begin{array}{l}150 . / 3 \\
150.09\end{array}$ & $\begin{array}{l}92.60 \\
92.20\end{array}$ \\
\hline FUNDACEP Horizonte & Fundacep & Precocious & 67.50 & b & 1904.30 & a & 108.81 & 143.18 & 87.96 \\
\hline TBIO Itaipu & Biotrigo & Medium & 74.85 & a & 1866.52 & a & 106.65 & 140.34 & 86.21 \\
\hline TBOO Pioneiro & Biotrigo & Medium & 67.64 & b & 1683.39 & b & 96.19 & 126.57 & 77.75 \\
\hline $\begin{array}{l}\text { TEC C219 } \\
\text { TEE } 10\end{array}$ & $\begin{array}{l}\text { CCGL-TEC } \\
\text { CCLLTEC }\end{array}$ & $\begin{array}{l}\text { Precocious } \\
\text { Medium }\end{array}$ & $\begin{array}{l}69.74 \\
6.821\end{array}$ & $\begin{array}{l}b \\
b \\
b\end{array}$ & $\begin{array}{l}1661.01 \\
15352\end{array}$ & b & $\begin{array}{l}94.91 \\
87.95\end{array}$ & $\begin{array}{l}124.89 \\
11560\end{array}$ & 76.72 \\
\hline $\begin{array}{l}\text { TEC C10 } \\
\text { TBIO Mestre }\end{array}$ & $\begin{array}{l}\text { CCCGLTECC } \\
\text { Biotrigo }\end{array}$ & $\begin{array}{l}\text { Medium } \\
\text { Medium }\end{array}$ & $\begin{array}{l}68.21 \\
68.29\end{array}$ & $\begin{array}{l}b \\
b \\
b\end{array}$ & $\begin{array}{l}1537.52 \\
1494.12\end{array}$ & $\begin{array}{c}b \\
b \\
b\end{array}$ & $\begin{array}{l}87.85 \\
85.38\end{array}$ & $\begin{array}{l}115.60 \\
112.34\end{array}$ & $\begin{array}{l}71.02 \\
69.01\end{array}$ \\
\hline FUNDACEP Cristalino & $\begin{array}{l}\text { Blortigo } \\
\text { Fundacep }\end{array}$ & $\begin{array}{l}\text { Mecalum } \\
\text { Precocious }\end{array}$ & $\begin{array}{l}68.29 \\
66.91\end{array}$ & $\begin{array}{l}\mathrm{b} \\
\mathrm{b}\end{array}$ & $\begin{array}{l}1443.412 \\
1436.15\end{array}$ & $\begin{array}{l}\mathrm{D} \\
\mathrm{c}\end{array}$ & $\begin{array}{l}85.06 \\
82.06\end{array}$ & $\begin{array}{l}112.34 \\
107.98\end{array}$ & $\begin{array}{l}69.01 \\
66.33\end{array}$ \\
\hline Ametista & OR Sementes & Precocious & 72.74 & a & 1416.02 & c & 80.91 & 106.47 & 65.40 \\
\hline Mirante & OR Sementes/Biotrigo & Precocious & 68.16 & b & 1352.38 & c & 77.28 & 101.68 & 62.47 \\
\hline $\begin{array}{l}\text { TBIOS Sinuelo } \\
\text { Média }\end{array}$ & Biotrigo & Medium & $\frac{62.83}{66.94}$ & & $\frac{1126.68}{1750.07}$ & $d$ & 64.38 & 84.71 & 52.04 \\
\hline Cultivars/2013 & Soure & cycle & $\begin{array}{ll}\mathrm{MH}^{-1 .} \\
\end{array}$ & & $\begin{array}{l}\text { Productivity } \\
\end{array}$ & & \%XE & \%XRS** & $\% \times B^{* * *}$ \\
\hline BRS 327 & EMBRAPA Trigo & Precocious & $\frac{10 \mathrm{~g} m i}{72.20}$ & $\mathrm{a}$ & 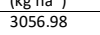 & $\mathrm{a}$ & 121.92 & 179.82 & 135.26 \\
\hline $\begin{array}{l}\text { Quartzo } \\
\text { R }\end{array}$ & OR Sementes/Biotrigo & Medium & 61.10 & & 2943.00 & $\mathrm{a}$ & 117.38 & 173.12 & 130.22 \\
\hline $\begin{array}{l}\text { TBIO Toruk } \\
\text { TBOO I Iuacu }\end{array}$ & $\begin{array}{l}\text { Biotrigo } \\
\text { Bistrijon }\end{array}$ & Medium & 61.80 & c & 2864.53 & a & 114.25 & 168.50 & 126.75 \\
\hline $\begin{array}{l}\text { TBOOI I vuacu } \\
\text { BRS Sarrudo }\end{array}$ & $\begin{array}{l}\text { Biotrigo } \\
\text { EMBRAPA Trigo }\end{array}$ & $\begin{array}{l}\text { Medium } \\
\text { Precocious }\end{array}$ & $\begin{array}{l}65.48 \\
66.4\end{array}$ & $\begin{array}{l}b \\
b \\
b\end{array}$ & $\begin{array}{l}2687.10 \\
2680.65\end{array}$ & $\begin{array}{l}b \\
b \\
b\end{array}$ & $\begin{array}{l}107.17 \\
109292\end{array}$ & $\begin{array}{l}158.06 \\
157.69\end{array}$ & $\begin{array}{l}118.90 \\
118.61\end{array}$ \\
\hline Ametista & OR Sementes & $\begin{array}{l}\text { Precocious } \\
\text { Pal }\end{array}$ & $\begin{array}{l}6.640 \\
68.20\end{array}$ & a & $\begin{array}{l}2680.65 \\
263.33 \\
\end{array}$ & b & $\begin{array}{l}105.92 \\
105.03 \\
\end{array}$ & $\begin{array}{l}157.69 \\
154.90\end{array}$ & $\begin{array}{l}118.61 \\
116.52\end{array}$ \\
\hline TEC 10 & CCGL-TEC & Medium & 66.25 & b & 2560.23 & b & 102.11 & $\begin{array}{l}154.90 \\
150.60\end{array}$ & 113.28 \\
\hline TBIO Mestre & Biotrigo & Medium & 65.73 & b & 2555.93 & b & 101.94 & 150.35 & 113.09 \\
\hline TBIO Sinuelo & $\begin{array}{l}\text { Biotrigo } \\
\text { Daf melhorament }\end{array}$ & $\begin{array}{l}\text { Medium } \\
\text { Medium }\end{array}$ & $\begin{array}{l}61.88 \\
6105\end{array}$ & c & 2550.53 & $\mathrm{~b}$ & 101.73 & 150.03 & 112.86 \\
\hline $\begin{array}{l}\text { DNAT ORO } \\
\text { TEC 6219 }\end{array}$ & $\begin{array}{l}\text { DNA mehoramento } \\
\text { CCGL-TEC }\end{array}$ & $\begin{array}{l}\text { Medium } \\
\text { Precocious }\end{array}$ & $\begin{array}{l}61.05 \\
7128\end{array}$ & c & $\begin{array}{l}2424.20 \\
239570\end{array}$ & $\mathrm{~b}$ & $\begin{array}{l}96.69 \\
9555\end{array}$ & 142.60 & $\begin{array}{l}107.27 \\
10600\end{array}$ \\
\hline TBIO Sintonia & Biotrigo & $\begin{array}{l}\text { Precocious } \\
\text { Precocious }\end{array}$ & $\begin{array}{l}1.1 .88 \\
69.83\end{array}$ & $\begin{array}{l}\mathrm{a} \\
\mathrm{a}\end{array}$ & $\begin{array}{l}2398.10 \\
2382.80\end{array}$ & $\begin{array}{l}\mathrm{b} \\
\mathrm{b}\end{array}$ & $\begin{array}{l}955.55 \\
95.04\end{array}$ & $\begin{array}{l}140.92 \\
140.16\end{array}$ & $\begin{array}{l}106.00 \\
10543\end{array}$ \\
\hline DNAT Prisma & DNA melhoramento & & 70.68 & a & 2319.25 & c & $\begin{array}{l}92.50 \\
92.50\end{array}$ & $\begin{array}{l}140.16 \\
136.43\end{array}$ & $\begin{array}{l}105.43 \\
102.62\end{array}$ \\
\hline BRS 331 & EMBRAPA Trigo & Super-precocious & 69.23 & $\mathrm{a}$ & 1920.43 & c & 76.59 & 112.97 & 84.97 \\
\hline $\begin{array}{l}\text { TEC Crontrale } \\
\text { Média }\end{array}$ & CCGL-TEC & Medium & $\frac{66.13}{66.48}$ & $\mathrm{~b}$ & $\begin{array}{l}\frac{1634.43}{2507.27} \\
\end{array}$ & $c$ & 65.19 & 96.14 & 72.32 \\
\hline Cultivars/2013 & Soure & Cycle & $\begin{array}{l}\mathrm{MH} \\
\left(\mathrm{kg} \mathrm{h}^{-1}\right)\end{array}$ & & $\begin{array}{l}\text { Productivity } \\
\left(\mathrm{kg} \mathrm{ha}^{-1}\right)\end{array}$ & & \%XE & \%XRS** & $\% \times \mathrm{BR}^{* *}$ \\
\hline DNAT ORO & DNA Melhoramento & Medium & (55.33) & ns & $4841.58^{*}$ & $\mathrm{~b}$ & 93.19 & 150.64 & 153.49 \\
\hline BRS Parrudo & EMBRAPA Trigo & Precocious & 76.85 & & 4831.32 & b & 92.99 & 150.32 & 152.17 \\
\hline Ametista & OR sementes & Precocious & 78.67 & & 4595.48 & b & 88.45 & 142.98 & 144.74 \\
\hline $\begin{array}{l}\text { BRS } 331 \\
\text { SCCroma }\end{array}$ & $\begin{array}{l}\text { EMBRAPA Trigo } \\
\text { Dafsermento }\end{array}$ & $\begin{array}{l}\text { Super-precocious } \\
\text { Precocius }\end{array}$ & $\begin{array}{l}78.12 \\
77.67\end{array}$ & & $\begin{array}{l}\begin{array}{l}4243.47 \\
4779.99\end{array} \\
\end{array}$ & b & $\begin{array}{l}81.72 \\
91.85\end{array}$ & $\begin{array}{l}132.09 \\
148.47\end{array}$ & $\begin{array}{l}133.72 \\
150.30\end{array}$ \\
\hline
\end{tabular}

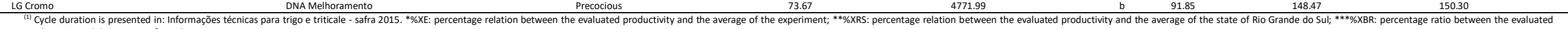
productivity and the average of Brazil; 


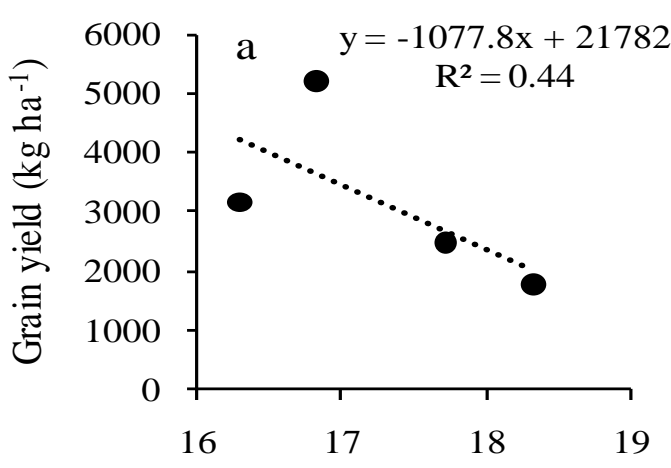

Avera ge tempera ture $\left({ }^{\circ} \mathrm{C}\right)$
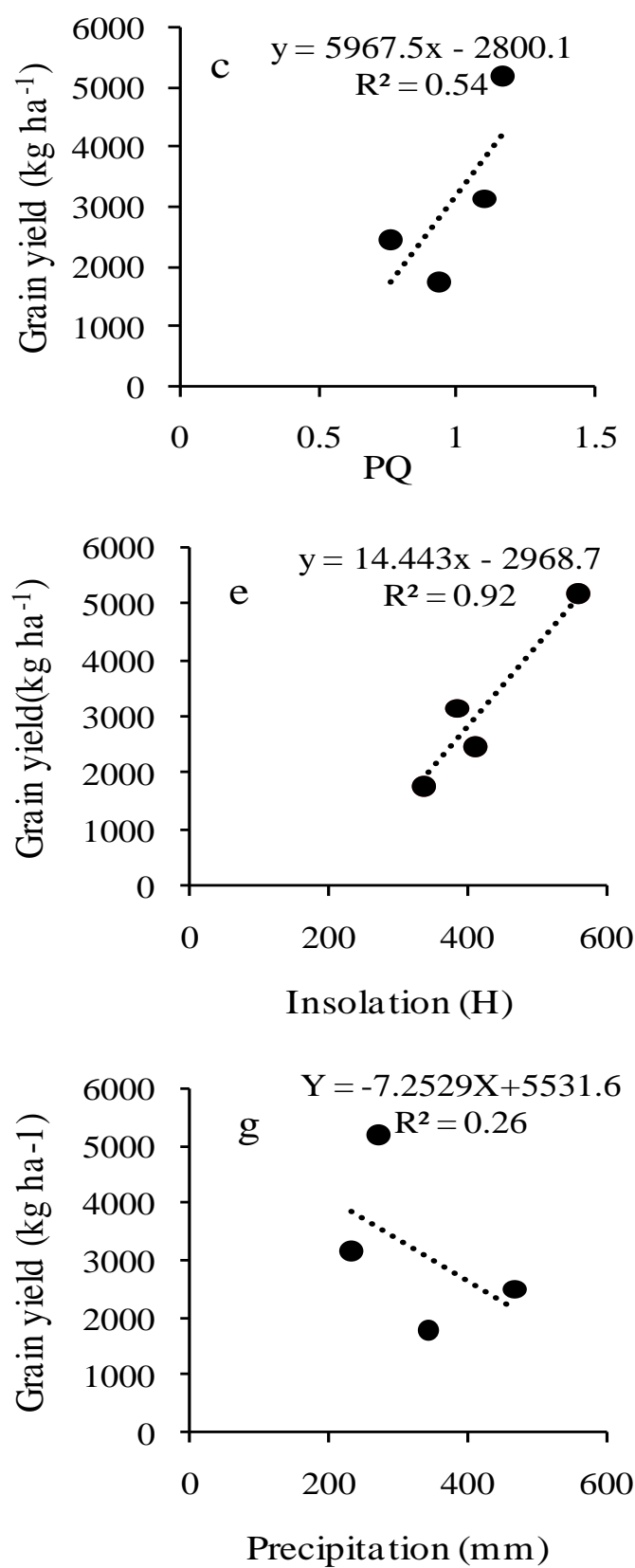

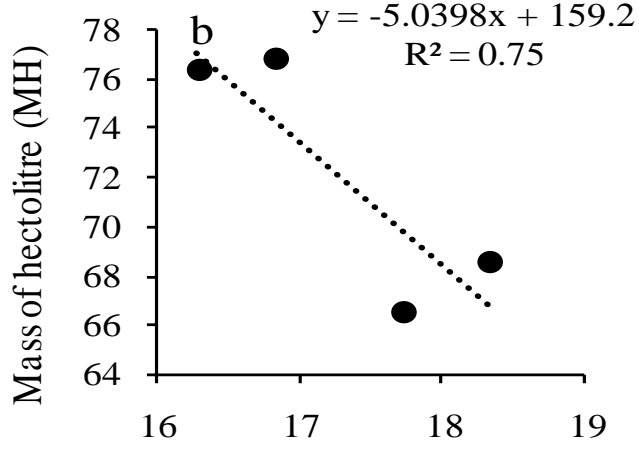

Average temperature $\left({ }^{\circ} \mathrm{C}\right)$
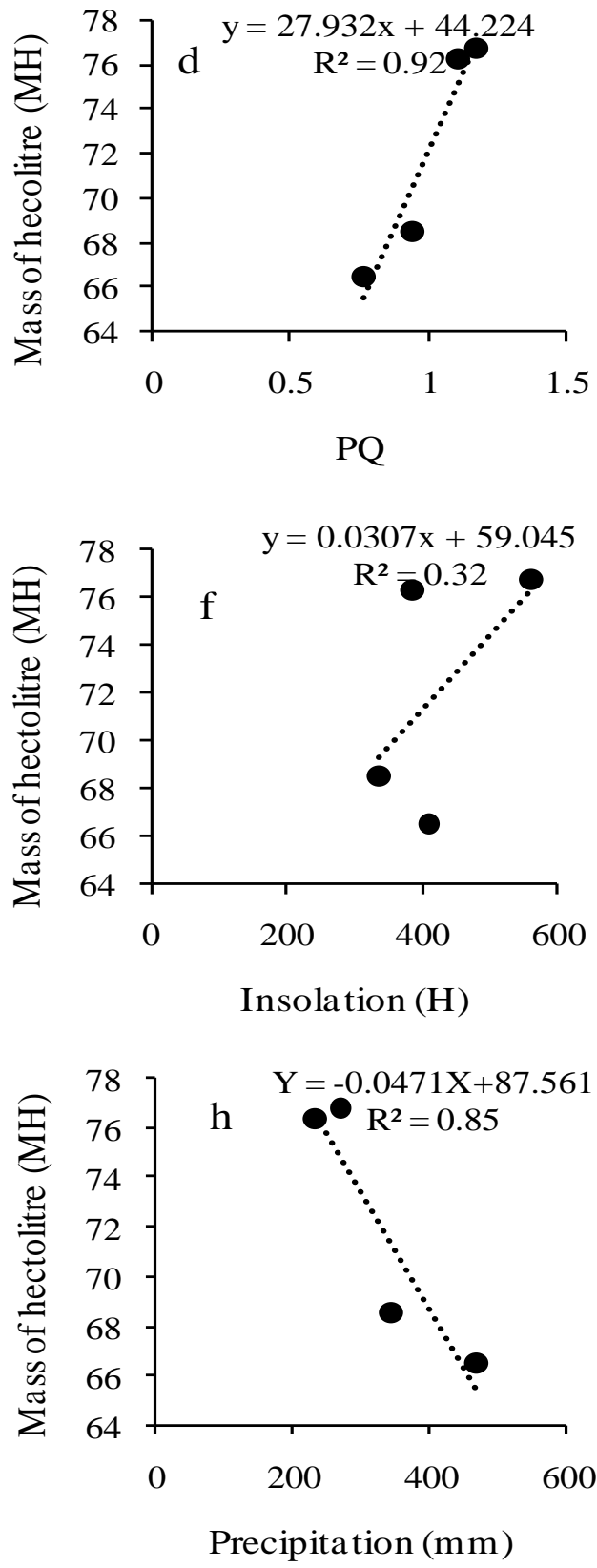

Fig 2. Mean of grain yield (PG) and hectoliter mass (MH) for each year of experiment (2013, 2014, 2015 and 2016) in relation to the average temperature of the growth period $(a, b)$ and the photothermal quotient $(c, d)$, insolation $(e, f)$ and precipitation in the period from 01/07 to 09/09. 
Table 3. Number of rainy days and accumulated rainfall in the cycle and during the maturation period for each agricultural year in Santa Maria.RS.

\begin{tabular}{lccc}
\hline $\begin{array}{l}\text { Agricultural } \\
\text { year }\end{array}$ & $\begin{array}{c}\text { Rainy } \\
\text { days }\end{array}$ & $\begin{array}{c}\text { Precipitation accumulated in the } \\
\text { cycle }(\mathrm{mm})\end{array}$ & $\begin{array}{c}\text { Precipitation accumulated in the last 30 days } \\
\text { of the cycle }(\mathrm{mm})\end{array}$ \\
\hline 2013 & 47 & 520.4 & 110.5 \\
2014 & 56 & 1119.1 & 215.9 \\
2015 & 42 & 931.1 & 484.4 \\
2016 & 40 & 710.8 & 385.4 \\
\hline
\end{tabular}

experiment had a yield of $1750.07 \mathrm{~kg} \mathrm{ha}^{-1}$, on average. According to Maddah et al. (2015) a rise in the predicted temperature due to global warming scenarios will definitely cause a decrease in the wheat yield, partly induced by the increase in the atmospheric $\mathrm{CO}_{2}$.

In contrary to the findings of this study, research in the literature indicates that while high temperatures affect the grain yield positively during the maturation period (Guarienti et al., 2004; Dreccer et al., 2018) they result in a loss in the hectoliter mass (Guarienti et al., 2004; Cargnin et al., 2006; Beillouin et al., 2018). While this action was not confirmed by the experiments in this study (Fig 2), the hectoliter mass was found to be affected by other environmental variables, like precipitation and the photothermal quotient during the time of anthesis.

Higher yields and $\mathrm{MH}$ values were characteristic of the years of 2013 and 2016, although a difference in the average yield of $1988.52 \mathrm{~kg} \mathrm{ha}^{-1}$ during these years was noteworthy. One of main reasons for this productivity difference is the utilization of new cultivars possessing greater productive potential. Cargnin (2006) reports that the average genetic gain in 30 years was $37 \mathrm{~kg} \mathrm{ha}^{-1}$ year ${ }^{-1}$, attributing to the improvement of cultivars used. Studies for the state of Rio Grande do Sul during the same period (2002 to 2013) identify a genetic progress in the grain yield of $61.36 \mathrm{~kg} \mathrm{ha}^{-1}$ year-1 $^{-1}$ (Follmann et al., 2017). In this context, it can be inferred that even though there is genetic gain with the adoption of new cultivars, the productivity difference between the agricultural years is largely affected by the meteorological factors.

Among the meteorological variables studied, FF revealed very close values for the average temperatures in two years. The main difference in the meteorological factors present during 2013 and 2016 is the number of hours of sun light, from $01 / 07$ to $30 / 09$, as 2016 was the year characterized by 178 hours of more available sunlight at this time of the year. From the recent studies on the effect of the photothermal coefficient it is clear that a direct relationship exists between this indicator and grain yield, assuming that all other resources are optimally available (Ahmed et al., 2010; Silva et al., 2014). The high number of available sunshine hours during this part of 2016 together with a lower average temperature enabled high productivity values. The International Maize and Wheat Improvement Center reported that canopy temperature is best evident during hot, sunny and cloudless days with low relative humidity $(\mathrm{RH}<60 \%)$ and warm air temperature (higher than $\left.15^{\circ} \mathrm{C}\right)$. It is known that conditions such as high vapor pressure deficit relate to several physiological factors like stomatal conductance, transpiration rate, plant water status, water use, leaf area index and crop yield (Pask et al., 2012).
Compared with 2014, the low insolation values was recorded for $01 / 07$ to $09 / 09$ and the high average temperature signified a low QF value. Besides, year 2014 revealed the highest rainfall. The combined effect of frequent rains and temperatures higher than normal supported the advent of significant plant diseases for the wheat cultivation in 2014. Interestingly, the year 2015 revealed that the QF values and those of the average temperatures exceed $17.7^{\circ} \mathrm{C}$, which were the key factors that limited the productivity. The years characterized by high precipitation (2014 and 2015) showed lowered $\mathrm{MH}$ and a linear relation, as the QF values from $01 / 07$ to $30 / 09$ were higher. The high precipitations in the reproductive and maturation phases boosted the grain metabolism, lowered the protein quality, and induced foliar stresses, which altered distribution of the photoassimilates, thus damaging the grain filling (Souza et al., 2013). During these four agricultural years of the experiments, other meteorological determinants were identified for yield and grain quality. They were distribution of precipitation during the cycle and the accumulation of precipitation during the maturation period (Table 3). In the year 2014 the crop experienced 56 days of rain, totally $1119.1 \mathrm{~mm}$ of accumulated rain. The high amount of accumulated precipitation, periods of cloudiness and high temperatures are the determinant factors that induced the low yields and low grain quality for this agricultural year. In 2015, the 42 days of rainfall accumulated $931.1 \mathrm{~mm}$ in total, during the cycle. In fact, $52 \%$ of this precipitation was received during the maturation period of the experiment ( 30 days prior to harvest), which is the cause for the low grain quality of this agricultural year. This excessive rainfall during the part of the harvest period significantly compromised the yield quality. Both 2014 and 2015 experienced the El Nino phenomenon, when the spring rains normally crossed the normal values. Cunha et al. (1999) reported that the years of the La Nina most favored the wheat cultivation in the region of Planalto, RS. In experiments simulating the irrigated winter wheat crops in the East of England under conditions of high temperatures, El Chami et al. (2015) confirmed that adequate irrigation distribution could facilitate up to $64 \%$ increase in grain yield. Wheat productivity results from interaction between the genetic and environmental factors. Therefore, management practices, including adaptation of the sowing window, supplementary irrigation and the cultivar positioning in accordance with the climatological predictions can be effective steps towards reducing the influence exerted by the extreme weather conditions on wheat output for the Santa Maria, RS, Brazil. 


\section{Materials and methods}

\section{Location discretion}

Four experiments were conducted during the agricultural years of 2013-2016 at the experimental site of the Crop Science Department of the Federal University of Santa Maria, with the following sowing and harvesting dates, respectively: 06/06/2013 and 06/11/2013;06/09/2014 and $11 / 17 / 2014 ; 06 / 03 / 2015$ and $10 / 18 / 2015$ and 06/16/2016 and $11 / 07 / 2016$. The cultivars listed in Table 2 , were used in the experiments arranged in a randomized complete block design with four replicates. The soil type was Red Argisol Dystrophic Arenico (Streck et al., 2008). This region experiences the type $C f a$ climate based on the Köppen classification (Heldwein et al., 2009). In the course of the experimental period, the meteorological variables (mean air temperature, solar radiation, insolation and accumulated precipitation) were recorded at the conventional meteorological station of the 8th Meteorology Department of the National Institute of Meteorology (DISME / INMET), situated roughly $1.4 \mathrm{~km}$ from the experimental site.

\section{Assessments}

The arithmetic mean of the maximum and minimum daily temperatures yielded the mean daily temperatures. The photothermal quotients (QF) (QF $=\mathrm{MJm}^{-2}$ day) from July 1 to September 30 of each agricultural year were assessed according to the methodology of Monteiro (2009): $Q=R / T$, where " $R$ " is the average daily solar radiation ( $\mathrm{MJm}^{-2}$ day), for the period between 1/07 and 30/09 of each agricultural year, and " $\mathrm{T}$ " is the average temperature for the same time period $\left({ }^{\circ} \mathrm{C}\right)$, subtracted from $4.5 \mathrm{O} \mathrm{C}$, which is the temperature base of the spring wheat in the phase approaching the anthesis (Monteiro, 2009). The management of the experiments was performed based on the technical indications for the wheat crop (Reunião, 2014), with the goal of inducing high yields. The harvests were conducted depending upon the physiological maturation of each cultivar. Humidity was adjusted to $13 \%$, with the dimensions of the useful area of the plot being equal to $15.75 \mathrm{~m}^{2}$. The grain productivity and hectoliter mass $(\mathrm{MH})$ of each cultivar were determined.

\section{Statistical analysis}

The Scott-Knott averages ( $\alpha \leq 0.05$ ) were tested using the SISVAR $^{\circledR}$ software (Ferreira, 2008).

\section{Conclusion}

The factors of solar radiation, availability and quality of sun hours (high photothermal quotient) and lower average air temperature are the determinant variables that govern the wheat productivity in the region of Santa Maria, RS. Regarding the hectoliter mass, the genetic effect exerts a greater influence following the lack of precipitation during the crop maturation phase and photothermal quotient around the time of anthesis.

\section{Acknowledgments}

The authors express their gratitude to the CNPQ for providing a research productivity grant to the second author and to CAPES for offering post graduate research grants to the other authors.

\section{References}

Ahmed M, Hussan F, Assim M, Aslam MA, Akram MN (2010) Correlation of photothermal quotient with spring wheat yield. Afr J Biot. 9(46):7869-7852.

Alberto CM, Streck NA, Walter LC, Rosa HT, Brackmann A, Oliveira FB, Zanon AJ, Fagundes LK (2009) Resposta à vernalização de cultivares brasileiras de trigo. Bragantia. 68(2): 535-543.

Beillouin D, Jeuffroy MH, Gauffreteau A (2018) Characterization of spatial and temporal combinations of climatic factors affecting yields: an empirical model applied to the French barley belt. Agr F Met. 262:402-411.

Cargnin A, de Souza MA, Carneiro PCS, Sofiatti V (2006) Interação entre genótipos e ambientes e implicações em ganhos com seleção em trigo. Pesq Agr Bras. 41(6):987993.

Conab - Companhia Nacional de Abastecimento (2016) Trigo-Brasil: séries históricas de área plantada, produtividade e produção, relativas às safras 1976 a 2016 de grãos, 2001 a 2014 de Café, 2005/06 a 2015/16 de cana-de-açúcar

Cunha GR, Dalmago GA, Estefanel V (1999) Enso influences on wheat crop in Brazil. Rev Bras Agrometeorol.7:127-138.

Dreccer MF, Fainges J, Whisha J, Ogbonnaya FC, Sadras VO (2018) Comparison of sensitive stages of wheat, barley, canola, chickpea and field pea to temperature and water stress across Australia. Agr For Met. 248(15):275-294.

El Chami D, Knox JW, Daccache A, Weatherhead EK (2015) The economics of irrigating wheat in humid climate - a study in the East of England. Agric Sys. 133:97-108.

Ferreira DF (2011) Sisvar: um programa para análises e ensino de estatística. Ciênc Agr.35(6):1039-1042.

Fischer RA (2016) The effect of duration of the vegetative phase in irrigated semi-dwarf spring wheat on phenology, growth and potential yield across sowing dates at low latitude. F C Res. 198:188-199.

Fischer RA (1985) Number of kernels in wheat crops and the influence of solar radiation and temperature. J Agric Sci. (Cambridge). 105(2):447-461.

Follmann DN, Cargnelutti Filho A, Lúcio AD, Souza VQ, Caraffa M, Wartha CA (2017) Genetic progress in homogeneous regions of wheat cultivation in Rio Grande do Sul State, Brazil. Gen Mol Res. 16(1):1-9.

Guarienti EM, Ciacco CF, Cunha GR, Del Duca LJA, Camargo CMO (2004) Influência das temperaturas mínima e máxima em características de qualidade industrial e em rendimento de grãos de trigo. Ciên T Aliment. 24(4):505515.

Heldwein AB, Buriol GA, Streck NA (2009) O clima de Santa Maria. Ciênc e Amb. 38(1):43-58.

Kumar PV, Rao VUM, Bhavani O, Dubey AP, Singh CB (2016) Effect of temperature and photothermal quotient on the yield components of wheat (Triticum aestivum L.) in IndoGangetic Plains of India. Exp Agri 52(1):14-35. 
Lizana XC, Calderini DF (2012) Yield and grain quality of wheat in response to increased temperatures at key periods for grain number and grain weight determination: considerations for the climatic change scenarios of Chile. J Agr Sci. 151(2):209-221.

Maddah V, Soltani A, Zeinali E, Bannayan-aval M (2015) Simulating climate change impacts on wheat production in Gorgan, Iran. Bull Env Pharm L Sci. 4(4):58-67.

Mapa- Ministério da Agricultura, Pecuária e Abastecimento (2017) Zoneamento Agrícola de Risco Climático para a cultura do Trigo no Estado do Rio Grande do Sul, ano-safra 2017. http://www.agricultura.gov.br (Accessed June 8, 2017).

Monteiro JE (2009) Agrometeorologia dos cultivos: o fator meteorológico na produção agrícola, 1rd edn. Brasília, DF, INMET. p 500.

Mota F (1998) Influência do clima sobre o rendimento de trigo no Rio Grande do Sul. Rev Bras Meteorol. 6(2):121124.

Nalley LL Barkley AP, Sayre K (2009) Photothermal quotient specifications to improve wheat cultivar yield componente models. Agron J. 101(3):556-563.

Pask A, Pietragalla J, Mullan D, Reynolds M (2012) Physiological breeding II: afield guide to wheat phenotyping. Mexico, DF, CIMMYT. p 132.

Porter JR, Gawith M (1999) Temperatures and the growth and development of wheat: a review. Eur J Agronomy. 10(1):23-36.
Ratjen AM, Böttcher ULF, Kage H (2012) Improved modeling of grain number in winter wheat. Field C R. 133:167-175

Reunião da Comissão Brasileira de Pesquisa de Trigo e Triticale, 8 (2014) Informações técnicas para trigo e triticale - safra 2015. Brasília, DF, Embrapa. p 229.

Scheeren PL, Caierão E, Silva MS, Del Luca LJA, Nascimento Junior A, Linhares A, Eicheberger L (2007) BRS Guamirim: cultivar de trigo da classe pão, precoce e de baixa estatura. Pesq Agrop Bras. 42(2): 293-296.

Semenov MA, Stratonovitch P, Alghabari F, Gooding MJ (2014) Adapting wheat in Europe for climate change. J Cer Sci. 59(3):245-256.

Silva RR, Benin G, Marchese JA, Silva EDB, Marchioro VS (2014) The use of photothermal quotient and frost risk to identify suitable sowing dates for wheat. Acta Sci Agron. 36(1):99-110.

Streck EV, Kampf N, Dalmolin RSD, Klamt E, Nascimento PC, Schneider P, Giasson E, Pinto LFS (2008) Solos do Rio Grande do Sul, 2rd edn. Porto Alegre, Emater. p 36.

Souza VQ, Nardino M, Bonato GO, Bahry CA, Caron BO, Zimmer PD, Schmidt D (2013) Desfolha em diferentes estádios fenológicos sobre características agronômicas em trigo. Biosc J. 29(6):1905-1911.

USDA - United Staates Departament of Agriculture. Crop Production. http://www.ers.usda.gov/dataproducts/wheat-data.aspx (Accessed January 26, 2016). 